\title{
Synthesis, Characterization and Anti Mycobacterial Activity of Novel Hydrazones
}

\author{
S KULANDAI THERESE* and G GEETHAMALIKA \\ Department of Chemistry, Nirmala College for Women, Coimbatore, Tamil Nadu, India. \\ ${ }^{*}$ Corresponding author E-mail: kulandaifspm @ gmail.com
}

http://dx.doi.org/10.13005/ojc/330140

(Received: December 23, 2016; Accepted: January 01, 2017)

\begin{abstract}
The hydrazone Schiff base analogues namely benzoic acid (4-allyloxy-benzylidene)-hydrazide and its series were designed and synthesized. The structures of newly synthesized compounds were characterized by analytical methods and spectral analysis and subjected to antibacterial and docking studies. The synthesized molecules were subjected to molecular docking studies using enoyl-acyl-carrier protein reductase (NADH) from mycobacterium tuberculosis as the receptor. The docking results confirm the binding affinity of the synthesized compounds with the selected receptor. Preliminary in-vitro anti bacterial studies were carried out with Gram-positive (Staphylococcus aureus) and Gram-negative (Escherichia coli) bacteria. Benzoic acid (4-allyloxy-benzylidene)-hydrazide was found to be most potent anti tuberculosis agent at 200 and $100 \mu \mathrm{g}$ per $\mathrm{ml}$, the percentage of inhibition was $98.7 \%$ and $97.9 \%$. The designed synthesized molecules were considered for evaluation for the molecular docking studies of their binding pattern with enoyl-acyl-carrier protein reductase from Mycobacterium tuberculosis. The antimycobacterial screening was performed against MTB H37Rv an isoniazid-resistant clinical isolate of MTB was used for the study.
\end{abstract}

Keywords: hydrazones, spectral studies, docking and anti mycobacterial activity.

\section{INTRODUCTION}

Tuberculosis (TB) is a worldwide pandemic caused by different species of mycobacteria. Mycobacterium tuberculosis is a successful pathogen, which causes tuberculosis and is the greatest single infectious cause of death worldwide, killing approximately two million people annually". Hydrazides are highly reactive bases with reducing properties. They have been used as a synthetic intermediate to produce different type of drugs in the treatment of different chronic illnesses. Hydrazides used in medicine include the anti-tuberculosis drug isoniazid and the antihypertensive and peripheral vasodilator drug hydralsane, whose hydrochloride is an effective drug for emergency reduction of blood pressure in hypertensive crisis. In the same way the hydrazine derivatives have been widely used in the 
treatment of tuberculosis and mental disorder. They can also be used as antimicrobial, antihypertensive, antimalarial and antitumoral agents ${ }^{2-11}$.

Schiff bases are considered to be among the most important group of compounds in medicinal chemistry due to their preparative accessibility, structural variety and wide biological profile ${ }^{12}$. The synthesized benzohydrazides were potent antimicrobial and anticancer agents ${ }^{13}$. Numbers of hydrazide-hydrazone derivatives possess antituberculosis activity ${ }^{14-21}$. The present study is aimed to carry out design, synthesis, in silico and in-vitro antimicrobial studies for the series of benzoic acid (4-allyloxy-benzylidene)-hydrazides.

\section{EXPERIMENTAL}

All the chemicals and solvents used are of analytical grade and were purchased from Royal scientific company and used without further purification. TLC was run on the silica coated aluminium sheets (TLC silica gel $60 \mathrm{~F}_{254}$, Analytical chromatography) and visualized in low UV light. IR spectra in $\mathrm{KBr}$ pellets were recorded on the FT-IR Perkin Elmer Spectrum spectrophotometer. ${ }^{1} \mathrm{H}$ NMR and $\mathrm{C}^{13} \mathrm{NMR}$ spectra were recorded on a $300 \mathrm{M} \mathrm{Hz}$ spectrometer with DMSO-d6 as solvent and TMS as internal reference. Chemical shifts are quoted as Sppm and the coupling constants $\mathrm{J}$ in $\mathrm{Hz}$ in signals are described as $s$ (singlet), d (doublet), t (triplet), $\mathrm{m}$ (multiplet) and $\mathrm{b}$ (broad). Melting points were determined on a Sigma melting point apparatus without corrections. GC MS mass spectrum was taken in JEOL GCMATE II GC-MS with Data system in a high resolution, double focusing instrument.

\section{General procedure for the synthesis of} benzohydrazides (3a-e)

The substituted benzoic acid $(2.46 \mathrm{~m} \mathrm{~mol})$ was refluxed with methanol in sulphuric acid for $4 \mathrm{~h}$. The ester formed reacts with hydrazine and gets converted to benzohydrazides by hydrozinolysis ${ }^{22}$.

Synthesis of Benzoic acid (4-allyloxybenzylidene)-hydrazide (5a-e)

Benzaldehyde undergoes nucleophilic addition reaction with aromatic hydrazides resulting in the formation of hydrazones. The completion of reaction and the homogeneity of the synthesized compounds were checked by TLC. Further the product formed was washed with petroleum ether, dried, recrystallized and characterized by spectral analysis.

Ethanolic solution of 4-allyloxy benzaldehyde (10 m mol) was added to a $10 \mathrm{~m} \mathrm{~mol}$ of ethanolic solution of benzohydrazides and the reaction mixture was stirred for 30 minutes at room temperature in the presence of glacial acetic acid as catalyst. After refluxing for 3-5 $\mathrm{h}$ the completion of the reaction was monitored by TLC. The reaction mixture was cooled and placed in refrigerator overnight. The resulting solid was filtered and washed with petroleum ether and recrystallised with ethanol.

\section{In silico Charaterisation}

The synthesized compounds were assessed in silico for their biological activity using PASS server $^{23}$. The three dimensional structure of the protein / enzymes was retrieved from Protein Data Bank $^{24}$. The compound under study was docked with the identified receptor using Autodock ${ }^{25}$ software and further analyzed for interactions using Pymol.

\section{Antimicrobial activity}

Antibacterial screening of the synthesized compounds was evaluated against Staphylococcus aureus and Escherichia coli, and the zone of inhibition was measured in $\mathrm{mm}$. The broth media was inoculated and grown at $37^{\circ} \mathrm{C}$ for $18 \mathrm{~h}$ to revive the bacteria from the stock cultures. The agar plates with wells were inoculated by spreading evenly with $18 \mathrm{~h}$ old bacterial cultures (100 $\mu \mathrm{l}, 10-4 \mathrm{cfu})$. After 20 min, various concentrations of the compound and the antibiotic were filled in the wells. Finally, the plates were incubated at $37^{\circ} \mathrm{C}$ for $24 \mathrm{~h}$ and the inhibition zone was observed ${ }^{26}$.

\section{Luciferase reporter phage (LRP) assay (27)}

The MTB H37Rv and clinical isolate of MTB were grown in Middlebrook 7H9 complete medium 12 with and without test sample for 3 days at $37^{\circ} \mathrm{C}$. LRP assay was done using concentrations of 200,100 and $50 \mu \mathrm{g} / \mathrm{mL}$ of test samples. $50 \mu \mathrm{L}$ bacterial suspension equivalent to MacFarlands. No. 2 standard was added to $400 \mu \mathrm{L}$ of $7 \mathrm{H} 9$ with and without the test compound. For each sample, two drug-free controls and two drug concentrations were prepared and this set up was incubated for 72 
$\mathrm{h}$ at $37^{\circ} \mathrm{C}$. After incubation, $50 \mu \mathrm{L}$ of the high titer luciferase reporter phage (phAETRC202) and 40 $\mu \mathrm{L}$ of $0.1 \mathrm{M} \mathrm{CaCl}_{2}$ were added to all the vials and this set up was incubated at $37^{\circ} \mathrm{C}$ for $4 \mathrm{~h}$. After incubation, $100 \mu \mathrm{L}$ of the mixture was taken from each tube into a Luminometer cuvette and an equal amount of working D-luciferin $(0.3 \mathrm{mM}$ in $0.05 \mathrm{M}$ sodium citrate buffer, $\mathrm{pH} 4.5$ ) solution was added. The Relative Light Units (RLU) was measured after 10 s of integration in the Luminometer. Readings were recorded in duplicate for each sample and the mean was calculated. The percentage reduction in the RLU was calculated for each test sample and compared with that of control.

\section{Broth microdilution method}

The MIC for MTB H37Rv and isoniazid (INH) resistant clinical isolate of MTB was determined using

Table 1: Activity prediction for the synthesized compounds

\begin{tabular}{llll}
\hline $\begin{array}{l}\text { Compound } \\
\text { code }\end{array}$ & Activity & Pa & Pi \\
\hline $5 a$ & Antituberculosic & 0.831 & 0.003 \\
$5 b$ & Antituberculosic & 0.827 & 0.003 \\
$5 c$ & Antituberculosic & 0.794 & 0.003 \\
$5 d$ & Antituberculosic & 0.781 & 0.003 \\
$5 e$ & Antituberculosic & 0.805 & 0.003 \\
\hline
\end{tabular}

a broth microdilution method (28) in Middlebrook 7H9 medium supplemented with OADC, with a final inoculum of $1 \times 10^{7} \mathrm{cfu} / \mathrm{mL}$. The compounds were dissolved in DMF $(1.25 \mathrm{mg} / \mathrm{mL})$ and used as stock solution. Concentrations ranging from 1250 to $1 \mu \mathrm{g} / \mathrm{mL}$ were used to assess the effectiveness of the compounds. After inoculation the microtiter tubes were incubated at $37^{\circ} \mathrm{C}$ for $72 \mathrm{~h}$, and the growth inhibition was recorded for 14 and 21 days, respectively. The MIC value represents the lowest dilution of the compound at which no bacterial growth was detected.

\section{RESULTS AND DISCUSSION}

Benzoic acid (4-allyloxy-benzylidene)hydrazide (5a) was prepared by the above mentioned method. benzohydrazide $(0.136 \mathrm{~g}$ $0.001 \mathrm{~mol} 1$ equivalent) 4-allyloxybenzaldehyde $(0.15 \mathrm{ml} 0.001 \mathrm{~mol} 1$ equivalent), pale yellow amorphous solid, yield: $90 \%$. m.p. $118-120^{\circ} \mathrm{C}$. FT-IR $\left(\mu_{\max } \mathrm{KBr}\right.$ pellets, $\left.\mathrm{cm}^{-1}\right)$ 935(N-N) str, 1256 (C-O-C), $1542(\mathrm{C}=\mathrm{C}), 1650(\mathrm{C}=\mathrm{N})$ str, $3032(\mathrm{C}-\mathrm{H})$ str. ${ }^{1} \mathrm{H}$ NMR $\left(\mathrm{CDCl}_{3}, 300 \mathrm{MHz}\right) \delta \mathrm{H}: 5.29-5.45(\mathrm{dd}, 1.2 \mathrm{~Hz}, 2 \mathrm{H})$, 5.9-6.1 $(\mathrm{m}, 1 \mathrm{H}), 4.57(\mathrm{~d}, 2 \mathrm{H}, 5.1 \mathrm{~Hz}), 6.9(\mathrm{~d}, 8.1 \mathrm{~Hz}$, $2 \mathrm{H}), 7.7(\mathrm{~d}, 6.6 \mathrm{~Hz}, 2 \mathrm{H}), 8.23(1 \mathrm{H}, \mathrm{s}), 9.2(1 \mathrm{H}, \mathrm{s}), 7.8$ (d, 6.6 Hz, 2H), 7.4-7.5 (m, 3H). ${ }^{13} \mathrm{C}$ NMR (DMSO $300 \mathrm{MHz}$ ): 160, 132, 131, 129, 128, 127, 126, 117, 114 and $68 . \mathrm{m} / \mathrm{z}=315(\mathrm{M}+1)$. Molecular Formula $\mathrm{C}_{17} \mathrm{H}_{16} \mathrm{~N}_{2} \mathrm{O}_{2}$

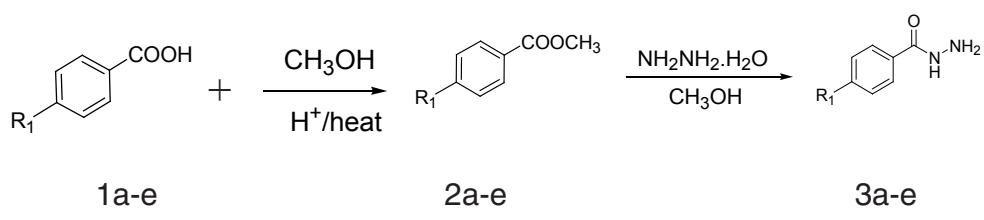

1a: $R 1=H, 1 b: R 1=C l, 1 c: R 1=C_{3}, 1 d: R 1=O_{3}$, 1e: $R 1=O H$

2a: $R 1=H, 2 b: R 1=C l, 2 c: R 1=C H_{3}, 2 d: R 1=O^{3}, 2 e: R 1=O H$ 3a: $R 1=H$, 3b: $R 1=C l$, 3c: $R 1=C_{3}$, 3d: $R 1=O_{3}$, 3e: $R 1=O H$

Scheme 1: Synthesis of Benzohydrazide from substituted benzoic acid

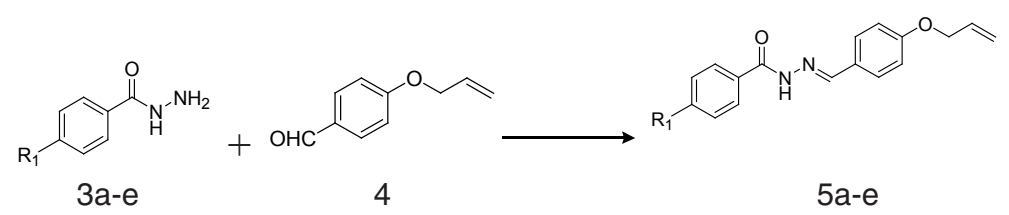

5a: R1 = H, 5b: R1 = Cl, 5c: R1 $=\mathrm{CH}_{3}, 5 \mathrm{~d}: \mathrm{R} 1=\mathrm{OCH}_{3}, 5 \mathrm{e}: \mathrm{R} 1=\mathrm{OH}$

Scheme 2: Synthesis of Benzoic acid (4-allyloxy-benzylidene)-hydrazides 


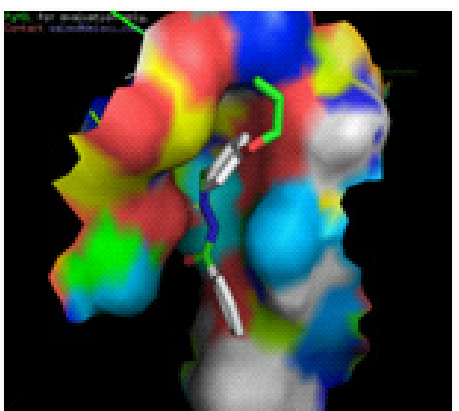

Fig 1: Binding interactions between Benzoic acid (4-allyloxy-benzylidene)hydrazide with the receptor

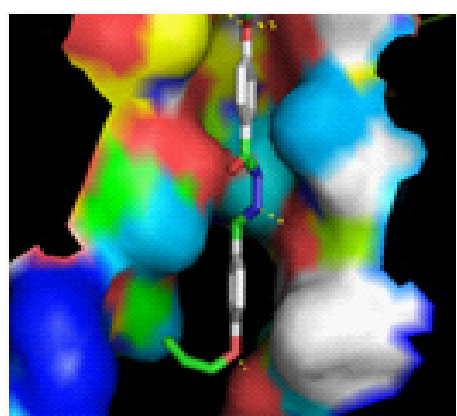

Fig 4: Binding interactions between 4-methoxy-benzoic acid (4-allyloxy-benylidene)hydrazide with the receptor

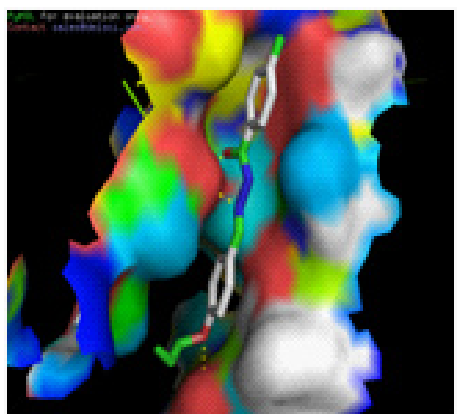

Fig 2: Binding interactions between 4-chloro-benzoic acid (4-allyloxy-benzylidene)hydrazide with the receptor

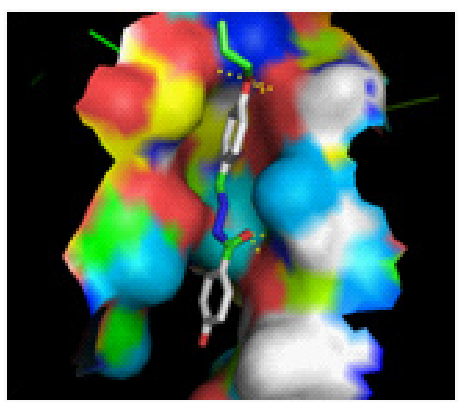

Fig 5: Binding interactions between 4- hydroxy -benzoic acid (4-allyloxy-benylidene)hydrazide with the receptor

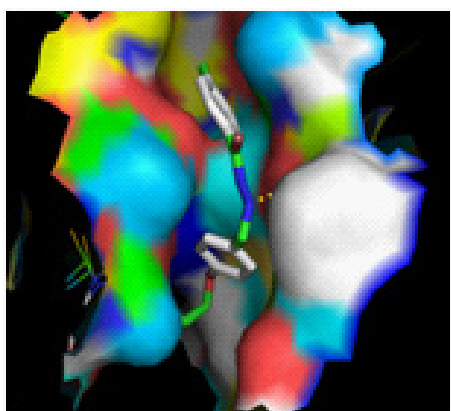

Fig 3: Binding interactions between 4-methyl-benzoic acid (4-allyloxy-benylidene)hydrazide with the receptor

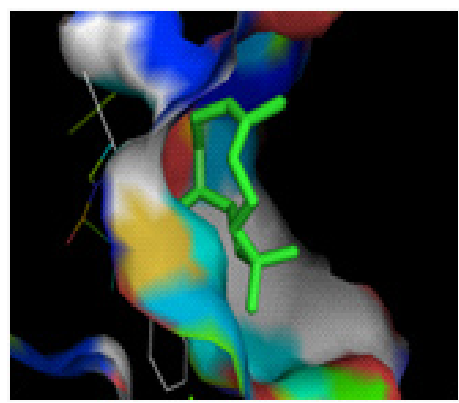

Fig 6: Binding interactions between isoniazid with the receptor

Table 2a: Docking results of Benzoic acid (4-allyloxy-benzylidene)-hydrazide with the receptor

\begin{tabular}{|c|c|c|c|c|c|c|c|c|c|c|c|}
\hline Pose & B.E & L.E & IC (nM) & Int.E & Vdw & Elec.E & Total.IE & Tor.E & Unb.E & $\begin{array}{c}\text { No. of } \\
\mathrm{H} \text { bonds }\end{array}$ & $\begin{array}{l}\text { Hydrogen } \\
\text { bond }\end{array}$ \\
\hline 1 & -11.81 & -0.56 & 2.22 & -13.6 & -13.59 & -0.01 & -0.92 & 1.79 & -0.92 & 1 & $\begin{array}{l}\text { Met98:HN: } \\
\text { :Lig1:O }\end{array}$ \\
\hline 2 & -10.79 & -0.51 & 12.37 & -12.58 & -12.58 & 0.0 & -0.81 & 1.79 & -0.81 & 0 & - \\
\hline 3 & -10.73 & -0.51 & 13 & -12.52 & -12.49 & -0.03 & & 1.79 & & 0 & - \\
\hline 4 & -10.72 & -0.51 & 13.94 & -12.51 & -12.52 & 0.01 & -1.03 & 1.79 & -1.03 & 0 & - \\
\hline 5 & -10.68 & -0.51 & 14.91 & -12.47 & -12.42 & -0.05 & -1.14 & 1.79 & -1.14 & 1 & $\begin{array}{l}\text { lle194:HN: } \\
\text { :Lig1:N }\end{array}$ \\
\hline 6 & -10.44 & -0.5 & 22.27 & -12.23 & -12.2 & -0.03 & -1.07 & 1.79 & -1.07 & 0 & - \\
\hline 7 & -10.33 & -0.49 & 26.91 & -12.12 & -12.04 & -0.07 & 00 & 1.79 & -0.95 & 1 & $\begin{array}{l}\text { Tyr158:HH: } \\
\text { :Lig1:O }\end{array}$ \\
\hline 8 & -9.84 & -0.47 & 61.29 & -11.63 & -11.62 & -0.01 & -0.85 & 1.79 & -0.85 & 2 & $\begin{array}{l}\text { Lig1:N: } \\
\text { :Ala201: } \\
\text { OLig1:N: } \\
\text { :Ala201:O }\end{array}$ \\
\hline 9 & -9.82 & -0.47 & 63.87 & -11.61 & -11.58 & -0.03 & -1.23 & 1.79 & -1.23 & 1 & $\begin{array}{l}\text { lle194:HN: } \\
\text { :Lig1:N }\end{array}$ \\
\hline 10 & -9.56 & -0.46 & 98.8 & -11.35 & -11.26 & -0.09 & -1.18 & 1.79 & -1.18 & 1 & $\begin{array}{l}\text { Tyr158:HH: } \\
\text { :Lig1:N }\end{array}$ \\
\hline
\end{tabular}


4-chloro-benzoic acid (4-allyloxybenzylidene)-hydrazide (5b) was prepared by the above mentioned method. 4-chlorobenzohydrazide $(0.171 \mathrm{~g} \quad 0.001 \mathrm{~mol} 1$ equivalent) 4-allyloxybenzaldehyde $(0.15 \mathrm{ml} 0.001 \mathrm{~mol}$ 1 equivalent), pale yellow amorphous solid, yield: $92 \%$ m.p. $158-160^{\circ} \mathrm{C}$. FT-IR $\left(v_{\max } \mathrm{KBr}\right.$ pellets, $\left.\mathrm{cm}^{-1}\right)$ 3434 or $3283(\mathrm{~N}-\mathrm{H}), 2930 \mathrm{Ar}(\mathrm{C}-\mathrm{H}) \mathrm{str}, 2849$ Ali $(\mathrm{CH})$ str, $1652(\mathrm{C}=\mathrm{O})$ str, $1506(\mathrm{C}=\mathrm{N})$ str, DMSO, 300 $\mathrm{MHz}) \delta \mathrm{H}: 5.2-5.3(\mathrm{~d}, \mathrm{~d}, 2 \mathrm{H} 1.5 \mathrm{~Hz}), 5.9-6.1(\mathrm{CH} 1 \mathrm{H}$ m), $4.63(\mathrm{~d} 2 \mathrm{H}, 5.1 \mathrm{~Hz}), 7.0(\mathrm{~d}, 2 \mathrm{H}, 8.7 \mathrm{~Hz}), 7.61(\mathrm{~d}$, $2 \mathrm{H}, 8.7 \mathrm{~Hz}), 5.3-5.4(\mathrm{~d}, \mathrm{~d} 1.5-1.8 \mathrm{~Hz}, 1 \mathrm{H}), 8.38(1 \mathrm{H}$, s), $7.9(\mathrm{~d}, 2 \mathrm{H}, 8.7 \mathrm{~Hz}), 7.6(\mathrm{~d}, 2 \mathrm{H}, 9 \mathrm{~Hz}) .{ }^{13} \mathrm{C}$ NMR (DMSO $300 \mathrm{MHz}$ ) 161, 159, 147 136, 133, 132, $129,128,128,117,115$ and $68 . \mathrm{m} / \mathrm{z}=315(\mathrm{M}+1)$. Molecular Formula $\mathrm{C}_{17} \mathrm{H}_{15} \mathrm{CIN}_{2} \mathrm{O}_{2}$. 4-methyl-benzoic acid (4-allyloxybenylidene)-hydrazide (5c) was prepared by the above mentioned method. 4-methyl benzohydrazide ( $0.15 \mathrm{~g} 0.001 \mathrm{~mol} 1$ equivalent) 4 -allyloxybenzaldehyde $(0.15 \mathrm{ml} 0.001 \mathrm{~mol} 1$ equivalent), yellow amorphous solid, yield: $88 \%$. m.p. 168ÚC. FT-IR $\left(v_{\max }\right.$ KBr pellets, $\left.\mathrm{cm}^{-1}\right) 937(\mathrm{~N}=\mathrm{N}), 1654(\mathrm{C}=\mathrm{O}), 1526(\mathrm{C}=\mathrm{N}) .{ }^{1} \mathrm{H}$ NMR (DMSO, $300 \mathrm{MHz}) \delta \mathrm{H}: 5.23-5.24(\mathrm{~d}, 2 \mathrm{H}), 5.89(\mathrm{~s}$, $1 \mathrm{H}), 4.61(2 \mathrm{H}), 6.8(\mathrm{~d}, 2 \mathrm{H}), 7.5(\mathrm{~d}, 2 \mathrm{H}), 8.1(\mathrm{~s}, 1 \mathrm{H})$, $8(\mathrm{~s}, 1 \mathrm{H}), 7.83(\mathrm{~d}, 2 \mathrm{H}), 7.24(\mathrm{~d}, 2 \mathrm{H}), 2.35(\mathrm{~S}, 3 \mathrm{H}) .{ }^{13} \mathrm{C}$ NMR (DMSO 300 MHz) 115, 137, 75, 164, 114, 130, $123,154,127,130.5,129,141$ and $20 . \mathrm{m} / \mathrm{z}=292$ $(\mathrm{M}+1)$. Molecular Formula $\mathrm{C}_{18} \mathrm{H}_{18} \mathrm{~N}_{2} \mathrm{O}_{2}$.

4-methoxy-benzoic acid (4-allyloxybenylidenehydrazide (5d) was prepared by the above mentioned method. 4-methoxy

Table 2b: Docking results of 4-chloro-benzoic acid (4-allyloxy-benzylidene)-hydrazide with the receptor

\begin{tabular}{|c|c|c|c|c|c|c|c|c|c|c|c|}
\hline Pose & e B.E & L.E & $\begin{array}{l}\text { IC } \\
(\mathrm{nM})\end{array}$ & Int.E & Vdw & $\begin{array}{c}\text { Elec. } \\
\text { E }\end{array}$ & $\begin{array}{l}\text { Total. } \\
\text { IE }\end{array}$ & Tor.E & $\begin{array}{c}\text { Unb. } \\
\text { E }\end{array}$ & $\begin{array}{c}\text { No. of } \\
H \text { bonds }\end{array}$ & Hydrogen bond \\
\hline 1 & -11.87 & -0.54 & 1.99 & -13.66 & -13.66 & -0.01 & -0.85 & 1.79 & -0.85 & 3 & $\begin{array}{l}\text { Lig1:O::4oim: } \\
\text { Ala201:OLig1:N: } \\
\text { :4oim:Ala201: } \\
\text { OLig1:N::4oim: } \\
\text { Ala201:O }\end{array}$ \\
\hline 2 & -11.31 & -0.51 & 5.09 & -13.1 & -13.11 & 0.01 & -0.81 & 1.79 & -0.81 & 3 & $\begin{array}{l}\text { Lig1:O::Gly205: } \\
\text { OLig1:N::Ala201 } \\
\text { :OLig1:N: } \\
\text { :Ala201:O }\end{array}$ \\
\hline 3 & -11.07 & -0.5 & 7.67 & -12.86 & -12.89 & 0.03 & -0.83 & 1.79 & -0.83 & 1 & Lig1:O::Gly96:O \\
\hline 4 & -11.04 & -0.5 & 8.12 & -12.83 & -12.86 & 0.04 & -0.79 & 1.79 & -0.79 & 3 & $\begin{array}{l}\text { Lig1:O::Ala201: } \\
\text { OLig1:O::Gly96: } \\
\text { OLig1:N: } \\
\text { :Met98:O }\end{array}$ \\
\hline 5 & -11.01 & -0.5 & 8.44 & -12.8 & -12.8 & 0.0 & -0.81 & 1.79 & -0.81 & 0 & - \\
\hline 6 & -10.54 & -0.48 & 18.78 & -12.33 & -12.34 & 0.01 & -0.63 & 1.79 & -0.63 & 1 & $\begin{array}{l}\text { Met98:HN: } \\
\text { :Lig1:O }\end{array}$ \\
\hline 7 & -10.51 & -0.48 & 19.66 & -12.3 & -12.36 & 0.05 & -0.54 & 1.79 & -0.54 & 0 & - \\
\hline 8 & -10.42 & -0.47 & 22.98 & -12.21 & -12.18 & -0.03 & -0.73 & 1.79 & -0.73 & 1 & Lig1:O::Ile194:O \\
\hline 9 & -9.86 & -0.45 & 58.91 & -11.65 & -11.63 & -0.02 & -0.8 & 1.79 & -0.8 & 1 & Lig1:N::Ala201:O \\
\hline 10 & -8.71 & -0.4 & 414.7 & -10.5 & -10.47 & -0.02 & -0.78 & 1.79 & -0.78 & 3 & $\begin{array}{l}\text { Ala157:HN::Lig1 } \\
\text { :N,NAsn 159: } \\
\text { HD21::Lig1: } \\
\text { OLig1:N: } \\
\text { :Asn159:OD1 }\end{array}$ \\
\hline
\end{tabular}


benzohydrazide $(0.166 \mathrm{~g} 0.001 \mathrm{~mol} 1$ equivalent) 4-allyloxybenzaldehyde $(0.15 \mathrm{ml} 0.001 \mathrm{~mol} 1$ equivalent), light yellow amorphous solid, yield: 88\%. m.p.162ÚC. FT-IR ( $v_{\max } \mathrm{KBr}$ pellets, $\left.\mathrm{cm}^{-1}\right)$ $1640(\mathrm{C}=\mathrm{N}), 937(\mathrm{~N}-\mathrm{N}), 1410$ (C-O), $2918 \operatorname{Ar}(\mathrm{C}-\mathrm{H})$. ${ }^{1} \mathrm{H}$ NMR (DMSO, $\left.300 \mathrm{MHz}\right) \delta \mathrm{H}: 5.23-5.24(\mathrm{~d}, 2 \mathrm{H}$ ), 5.89(s, $1 \mathrm{H}), 4.61(2 \mathrm{H}), 6.8(\mathrm{~d}, 2 \mathrm{H}), 7.5(\mathrm{~d}, 2 \mathrm{H}), 8.1(\mathrm{~s}$, $1 \mathrm{H}), 8(\mathrm{~s}, 1 \mathrm{H}), 7.84(\mathrm{~d}, 2 \mathrm{H}), 6.95(\mathrm{~d}, 2 \mathrm{H}), 3.73(\mathrm{~s}, 3 \mathrm{H})$. ${ }^{13} \mathrm{C}$ NMR (DMSO $300 \mathrm{MHz}$ ) 115, 137, 75, 164, 114, $130,123,154,125,128,114,165$ and 56. $\mathrm{m} / \mathrm{z}=292$ $(\mathrm{M}+1)$. Molecular Formula $\mathrm{C}_{18} \mathrm{H}_{18} \mathrm{~N}_{2} \mathrm{O}_{2}$.

4- hydroxy -benzoic acid (4-allyloxybenzylidene)- hydrazide (5e) was prepared by the above mentioned method. 4-hydroxy benzohydrazide $(0.152 \mathrm{~g} 0.001 \mathrm{~mol} 1$ equivalent) 4-allyloxybenzaldehyde $(0.15 \mathrm{ml} 0.001 \mathrm{~mol} 1$ equivalent), yellow amorphous solid, yield: $84 \%$. m.p. 178ÚC. FT-IR $\left(v_{\max } \mathrm{KBr}\right.$ pellets, $\left.\mathrm{cm}^{-1}\right) 1535$ $(\mathrm{C}=\mathrm{C}), 1625(\mathrm{C}=\mathrm{N}), 3250(\mathrm{~N}-\mathrm{H}), 1658(\mathrm{C}=\mathrm{O}) .{ }^{1} \mathrm{H}$ NMR (DMSO, $300 \mathrm{MHz}) \delta \mathrm{H}: 5.23-5.24(\mathrm{~d}, 2 \mathrm{H})$, 5.89(s, $1 \mathrm{H}), 4.61(2 \mathrm{H}), 6.8(\mathrm{~d}, 2 \mathrm{H}), 7.5(\mathrm{~d}, 2 \mathrm{H}), 8.1(\mathrm{~s}$, $1 \mathrm{H}), 8(\mathrm{~s}, 1 \mathrm{H}), 7.78(\mathrm{~d}, 2 \mathrm{H}), 6.91(\mathrm{~d}, 2 \mathrm{H}), 5(\mathrm{~S}, 1 \mathrm{H})$. ${ }^{13} \mathrm{C}$ NMR (DMSO $300 \mathrm{MHz}$ ) 115, 137, 75, 164, 114, 130, 123, 154, 126, 128, 115 and $160 . \mathrm{m} / \mathrm{z}=310$ $(\mathrm{M}+1)$. Molecular Formula $\mathrm{C}_{18} \mathrm{H}_{18} \mathrm{~N}_{2} \mathrm{O}_{3}$.
Synthesized compounds (Benzoic acid (4-allyloxy-benzylidene)-hydrazide), (4-chlorobenzoic acid (4-allyloxy-benzylidene)-hydrazide), were subjected to a preliminary anti bacterial screening against the strain gram positive (Staphylococcus aureus) and Gram-negative (Escherichia coll) bacteria to assess the antibacterial susceptibility of the test compounds. The compounds showed good inhibition at lower concentration.

It is evident from table-1 that the compound 5 a exhibits maximum anti-tuberculosis activity among all the study compounds as its probability of being active $(\mathrm{Pa})$ value is 0.831 and Probability of being inactive $(\mathrm{Pi})$ is only 0.003 . Also all the other compounds demonstrate anti-tuberculosic activity with $\mathrm{Pa}$ between 0.827 and 0.781 . Hence, all the study compounds were docked with the target Enoyl-[acyl-carrier-protein] reductase [NADH] from Mycobacterium tuberculosis. The interaction of the synthesized compound with the target was evaluated using Docking studies. The docking scores along with the other parameters for the different poses of the docked molecules are presented in Tables 2(a) $-2(e)$.

Table 2c: Docking results of 4-methyl-benzoic acid (4-allyloxy-benylidene)- hydrazide with the receptor

\begin{tabular}{|c|c|c|c|c|c|c|c|c|c|c|c|}
\hline Pose & B.E & L.E & $\begin{array}{c}\text { IC } \\
(\mathrm{nM})\end{array}$ & Int.E & Vdw & $\begin{array}{c}\text { Elec. } \\
\text { E }\end{array}$ & $\begin{array}{l}\text { Total. } \\
\text { IE }\end{array}$ & Tor.E & Unb.E & $\begin{array}{c}\text { No. of } \\
\mathrm{H} \text { bonds }\end{array}$ & $\begin{array}{l}\text { Hydrogen } \\
\text { bond }\end{array}$ \\
\hline 1 & -11.37 & -0.52 & 4.64 & -13.46 & -13.48 & 0.03 & -0.8 & 2.09 & -0.8 & 2 & $\begin{array}{l}\text { LIG1:N: } \\
\text { :MET98:OLIG1 } \\
\text { :N::MET98:O }\end{array}$ \\
\hline 2 & -11.17 & -0.51 & 6.48 & -13.26 & -13.25 & -0.01 & -0.85 & 2.09 & -0.85 & 0 & - \\
\hline 3 & -10.69 & -0.49 & 14.59 & -12.78 & -12.78 & 0.0 & -0.85 & 2.09 & -0.85 & 0 & - \\
\hline 4 & -10.61 & -0.48 & 16.73 & -12.7 & -12.68 & -0.02 & -0.91 & 2.09 & -0.91 & 2 & $\begin{array}{l}\text { LIG1:O: } \\
\text { :MET98: } \\
\text { OMET98:HN: } \\
\text { :LIG1:O }\end{array}$ \\
\hline 5 & -10.4 & -0.47 & 23.61 & -12.49 & -12.47 & -0.03 & -0.92 & 2.09 & -0.92 & 0 & - \\
\hline 6 & -10.3 & -0.47 & 28.01 & -12.39 & -12.34 & -0.05 & -0.83 & 2.09 & -0.83 & 1 & $\begin{array}{l}\text { ILE194:HN: } \\
\text { :LIG1:O }\end{array}$ \\
\hline 7 & -10.02 & -0.46 & 45.14 & -12.11 & -12.08 & -0.02 & -0.94 & 2.09 & -0.94 & 0 & - \\
\hline 8 & -9.98 & -0.45 & 48.37 & -12.07 & -12.01 & -0.05 & -1.09 & 2.09 & -1.09 & 1 & $\begin{array}{l}\text { TYR158:HH: } \\
\text { :LIG1:O }\end{array}$ \\
\hline 9 & -9.74 & -0.44 & 72.73 & -11.83 & -11.72 & -0.11 & -1.05 & 2.09 & -1.05 & 0 & - \\
\hline 10 & -9.7 & -0.44 & 77.03 & -11.79 & -11.76 & -0.03 & -0.94 & 2.09 & -0.94 & 0 & - \\
\hline
\end{tabular}


The docking results of Enoyl-[acyl-carrierprotein] reductase [NADH] from Mycobacterium tuberculosis with the ligand Benzoic acid (4-allyloxybenzylidene)-hydrazide is presented in table $2 a$. The binding energies of the different binding conformations range between -11.81 and $-9.56 \mathrm{kcal} /$ mol. Among the ten different docked conformations conformation 8 had a binding energy of $-9.84 \mathrm{kcal} /$ mol with 2 hydrogen bonds between the ligand Benzoic acid (4-allyloxy-benzylidene)-hydrazide and Ala 201. The conformation 1, Conformation 5, Conformation 7, Conformation 9 and Conformation 10 had only one hydrogen bond formed with binding energies -11.81, -10.68, -10.33, -9.82 and -9.56 $\mathrm{kcal} / \mathrm{mol}$ respectively. Hence, conformation 8 with 2 hydrogen bonds and binding energy of $-9.84 \mathrm{kcal} /$ $\mathrm{mol}$ is considered the best docked conformation and represented in figure-1
The table $2 \mathrm{~b}$ presents the ten different binding conformations of Enoyl-[acyl-carrier-protein] reductase [NADH] from Mycobacterium tuberculosis with the ligand 4-chloro-benzoic acid (4-allyloxybenzylidene)-hydrazide. The binding energy ranges from $-11.87 \mathrm{kcal} / \mathrm{mol}$ to $-8.71 \mathrm{kcal} / \mathrm{mol}$. The conformations 1,2,4 and 10 had three hydrogen bonds between the receptor, Enoyl-[acyl-carrierprotein] reductase [NADH] and ligand 4-chlorobenzoic acid (4-allyloxy-benzylidene)-hydrazide. In the first binding conformation, all the three hydrogen bonds are formed between the ligand and Alanine 201 in Enoyl-[acyl-carrier-protein] reductase [NADH] protein. But in the second binding conformation two hydrogen bonds are formed between the ligand and Alanine 201 and the third hydrogen bond is formed between the ligand and Glycine 205 in the receptor. Alternatively in the binding conformation 4, Alanine 201, Glycine 96 and Methionine 98 in the receptor

Table 2d: Docking results of 4-methoxy-benzoic acid (4-allyloxy-benylidene)- hydrazide with the receptor

\begin{tabular}{|c|c|c|c|c|c|c|c|c|c|c|c|}
\hline Pose & B.E & L.E & $\begin{array}{c}\text { IC } \\
(\mathrm{nM})\end{array}$ & Int.E & Vdw & $\begin{array}{c}\text { Elec. } \\
\text { E }\end{array}$ & $\begin{array}{l}\text { Total. } \\
\text { IE }\end{array}$ & Tor.E & Unb.E & $\begin{array}{c}\text { No. of } \\
\mathrm{H} \text { bonds }\end{array}$ & $\begin{array}{l}\text { Hydrogen } \\
\text { bond }\end{array}$ \\
\hline 1 & -11.89 & -0.52 & 1.93 & -13.98 & -13.97 & -0.01 & -0.85 & 2.09 & -0.85 & 1 & $\begin{array}{l}\text { LIG1:N: } \\
\text { :ALA201:O }\end{array}$ \\
\hline 2 & -10.96 & -0.48 & 9.29 & -13.05 & -13.09 & 0.04 & -0.82 & 2.09 & -0.82 & 1 & $\begin{array}{l}\text { LIG1:O: } \\
\text { :PRO156:O }\end{array}$ \\
\hline 3 & -10.89 & -0.47 & 10.41 & -12.98 & -12.98 & 0.0 & -0.85 & 2.09 & -0.85 & 2 & $\begin{array}{l}\text { LIG:N::ALA201: } \\
\text { OLIG1:N: } \\
\text { :ALA201:O }\end{array}$ \\
\hline 4 & -10.8 & -0.47 & 12.02 & -12.89 & -12.89 & 0.0 & -0.91 & 2.09 & -0.91 & 4 & $\begin{array}{l}\text { LIG1:N::ALA201 } \\
\text { :OLIG1:O: } \\
\text { :ASN106: } \\
\text { OD1LIG1:O: } \\
\text { :GLY96:OLIG1: } \\
\text { O::ALA201:O }\end{array}$ \\
\hline 5 & -10.62 & -0.46 & 16.4 & -12.71 & -12.67 & -0.03 & -0.87 & 2.09 & -0.87 & 0 & - \\
\hline 6 & -10.41 & -0.45 & 23.36 & -12.5 & -12.46 & -0.04 & -0.93 & 2.09 & -0.93 & 1 & $\begin{array}{l}\text { LIG1:O: } \\
\text { :MET98:O }\end{array}$ \\
\hline 7 & -10.16 & -0.44 & 35.45 & -12.25 & -12.27 & 0.02 & -0.73 & 2.09 & -0.73 & 1 & $\begin{array}{l}\text { LIG1:O: } \\
\text { :PRO156:O }\end{array}$ \\
\hline 8 & -9.7 & -0.42 & 77.07 & -11.79 & -11.67 & -0.12 & -0.84 & 2.09 & -0.84 & 1 & $\begin{array}{l}\text { MET98:HN: } \\
: \text { LIG1:O }\end{array}$ \\
\hline 9 & -9.41 & -0.41 & 125.97 & -11.5 & -11.46 & -0.04 & -0.9 & 2.09 & -0.9 & 1 & $\begin{array}{l}\text { LIG1:O: } \\
\text { :ALA201:O }\end{array}$ \\
\hline 10 & -8.98 & -0.39 & 259.88 & -11.07 & -10.94 & -0.13 & -0.85 & 2.09 & -0.85 & 0 & - \\
\hline
\end{tabular}


form one hydrogen bond each with the ligand. Finally, the conformation 10 had 3 hydrogen bonds formed each between Alanine 157, Asparagine 159, Asparagine 159 and the ligand with a binding energy of $-8.71 \mathrm{kcal} / \mathrm{mol}$. Among the ten different binding conformations, the conformation 1 with the least binding energy and three hydrogen bonds is chosen as the best possible orientation which is displayed in Figure-2

The table $2 \mathrm{c}$ depicts the different binding orientations of the ligand $\mathrm{K} 1 \mathrm{~b}$ with the receptor

Table 2e: Docking results of 4- hydroxy -benzoic acid (4-allyloxy-benylidene)- hydrazide with the receptor

\begin{tabular}{|c|c|c|c|c|c|c|c|c|c|c|c|}
\hline Pose & B.E & L.E & $\begin{array}{c}\text { IC } \\
(n M)\end{array}$ & Int.E & Vdw & $\begin{array}{c}\text { Elec. } \\
\text { E }\end{array}$ & $\begin{array}{l}\text { Total. } \\
\text { IE }\end{array}$ & Tor.E & Unb.E & $\begin{array}{c}\text { No. of } \\
\mathrm{H} \text { bonds }\end{array}$ & $\begin{array}{l}\text { Hydrogen } \\
\text { bond }\end{array}$ \\
\hline 1 & -11.35 & -0.52 & 4.83 & -13.14 & -13.12 & -0.02 & -0.61 & 1.79 & -0.61 & 1 & $\begin{array}{l}\text { LIG:N: } \\
\text { :ALA201:O }\end{array}$ \\
\hline 2 & -10.26 & -0.47 & 30.34- & -12.05 & -12.03 & -0.02 & -0.81 & 1.79 & -0.81 & 0 & - \\
\hline 3 & -10.25 & -0.47 & 30.41 & -12.04 & -12.08 & 0.03 & -0.81 & 1.79 & -0.81 & 0 & - \\
\hline 4 & 9.97 & -0.45 & 49.19 & -11.76 & -11.74 & -0.02 & -0.58 & 1.79 & -0.58 & 0 & - \\
\hline 5 & -9.95 & -0.45 & 50.58 & -11.74 & -11.74 & -0.01 & -0.64 & 1.79 & -0.64 & 2 & $\begin{array}{l}\text { LIG1:N::ALA201 } \\
\text { :OLIG1:O: } \\
\text { :ASN106:OD1 }\end{array}$ \\
\hline 6 & -9.78 & -0.44 & 67.28 & -11.57 & -11.59 & 0.02 & -0.73 & 1.79 & -0.73 & 0 & - \\
\hline 7 & -9.76 & -0.44 & 70.34 & -11.55 & -11.62 & 0.07 & -0.79 & 1.79 & -0.79 & 1 & LIG:O::MET98:O \\
\hline 8 & -9.74 & -0.44 & 72.95 & -11.53 & -11.49 & -0.04 & -0.77 & 1.79 & -0.77 & 2 & $\begin{array}{l}\text { LIG1:O::MET98: } \\
\text { OMET98:HN: } \\
\text { :LIG1:O }\end{array}$ \\
\hline 9 & -9.73 & -0.44 & 73.19 & -11.52 & -11.53 & 0.01 & -0.84 & 1.79 & -0.84 & 1 & $\begin{array}{l}\text { LIG1:N: } \\
\text { :ALA201:O }\end{array}$ \\
\hline 10 & -7.1 & -0.32 & 6.29 & -8.89 & -8.84 & -0.05 & -0.77 & 1.79 & -0.77 & 0 & - \\
\hline
\end{tabular}

Table 2f: Docking results of isoniazid with the receptor

\begin{tabular}{|c|c|c|c|c|c|c|c|c|c|c|c|}
\hline Pose & B.E & L.E & $\begin{array}{c}\text { IC } \\
(n M)\end{array}$ & Int.E & Vdw & $\begin{array}{c}\text { Elec. } \\
\text { E }\end{array}$ & $\begin{array}{l}\text { Total. } \\
\text { IE }\end{array}$ & Tor.E & Unb.E & $\begin{array}{c}\text { No. of } \\
\mathrm{H} \text { bonds }\end{array}$ & $\begin{array}{l}\text { Hydrogen } \\
\text { bond }\end{array}$ \\
\hline 1 & -6.73 & -0.67 & 11.61 & -7.03 & -7.0 & -0.04 & -0.09 & 0.3 & -0.09 & 1 & Iso:O::Ile $202: 0$ \\
\hline 2 & -6.73 & -0.67 & 11.69 & -7.03 & -6.99 & -0.04 & -0.09 & 0.3 & -0.09 & 1 & Iso:O::Ile $202: 0$ \\
\hline 3 & -6.71 & -0.67 & 12.11 & -7.01 & -6.97 & -0.03 & -0.09 & 0.3 & -0.09 & 1 & Iso:O::Ile $202: 0$ \\
\hline 4 & -6.67 & -0.67 & 12.94 & -6.97 & -6.98 & 0.01 & -0.09 & 0.3 & -0.09 & 1 & Iso:O::Ala 201:O \\
\hline 5 & -6.67 & -0.67 & 13.01 & -6.96 & -6.98 & 0.02 & -0.09 & 0.3 & -0.09 & 1 & Iso:O::Ala 201:O \\
\hline 6 & -6.54 & -0.65 & 16.15 & -6.84 & -6.73 & -0.11 & -0.09 & 0.3 & -0.09 & 0 & \\
\hline 7 & -6.04 & -0.60 & 37.35 & -6.34 & -6.26 & -0.08 & -0.09 & 0.3 & -0.09 & 2 & $\begin{array}{l}\text { Tyr158:HH::Iso:O } \\
\text { Ile194:HN::Iso:N }\end{array}$ \\
\hline 8 & -6.04 & -0.60 & 37.62 & -6.33 & -6.25 & -0.08 & -0.09 & 0.3 & -0.09 & 2 & $\begin{array}{l}\text { Tyr158:HH::Iso: } \\
\text { Olle194:HN: } \\
\text { :Iso:N }\end{array}$ \\
\hline 9 & -6.00 & -0.60 & 39.78 & -6.30 & -6.21 & -0.09 & -0.09 & 0.3 & -0.09 & 1 & Ile194:HN::Iso:N \\
\hline 10 & -5.83 & -0.58 & 53.14 & -6.13 & -6.06 & -0.07 & -0.09 & 0.3 & -0.09 & 1 & Ile194:HN::Iso:O \\
\hline
\end{tabular}


Table 3: Antimicrobial activity data of $5 a$ and $5 b$ by LPR assay

\begin{tabular}{lccc}
\hline $\begin{array}{l}\text { Comp. } \\
\text { code }\end{array}$ & $\begin{array}{c}\text { Conc. } \\
(\mathrm{mL})\end{array}$ & $\begin{array}{c}\text { CFU } \\
(\mathrm{col} / \mathrm{mL})\end{array}$ & $\begin{array}{c}\% \\
\text { inhibition }\end{array}$ \\
\hline $5 \mathrm{a}$ & 10 & $3.2 \times 10^{6}$ & 19.1 \\
& 20 & & 0 \\
& 50 & & 0 \\
& 200 & & \\
$5 \mathrm{~b}$ & 100 & $4.4 \times 10^{6}$ & 97.9 \\
& 200 & $2.4 \times 10^{6}$ & 98.7 \\
& 400 & & - \\
Control-1 & - & $5.8 \times 10^{6}$ & - \\
Control-2 & - & $5.0 \times 10^{6}$ & \\
\hline
\end{tabular}

Control-1 -H37Rv

control-2 -H37Rv

Enoyl-[acyl-carrier-protein] reductase [NADH] from Mycobacterium tuberculosis. Poses 1 and 4 have two hydrogen bonds formed by ' $\mathrm{O}$ ' of Met98 and ' $\mathrm{N}$ ' and ' $O$ ' atoms in the ligand. Poses 6 and 8 had one hydrogen bond each between ' $H$ ' atom of Ile 194 and Tyr 158 respectively with the 'O' atom of the Ligand. Among the ten different binding conformations, the conformation 1 with binding energy of $-11.37 \mathrm{kcal} /$ $\mathrm{mol}$ and two hydrogen bonds is chosen as the best possible orientation which is displayed in Figure 3.

The table $2 \mathrm{~d}$ presents the ten different binding conformations of Enoyl-[acyl-carrier-protein] reductase $[\mathrm{NADH}]$ from Mycobacterium tuberculosis with the ligand $5 \mathrm{~d}$. The binding energy ranges from $-11.89 \mathrm{kcal} / \mathrm{mol}$ to $-8.98 \mathrm{kcal} / \mathrm{mol}$. The poses 1 , 2, 6, 7, 8 and 9 had one hydrogen bond between the receptor, Enoyl-[acyl-carrier-protein]reductase [NADH] and ligand $5 \mathrm{~d}$. But pose 3 and 4 had 2 and four hydrogen bonds between the receptor and the ligand. Hence, among the ten different binding conformations, the conformation 4 with the binding energy of -10.8 and 4 hydrogen bonds is chosen as the best possible orientation which is displayed in Figure 4.

The table $2 \mathrm{e}$ presents the ten different binding conformations of Enoyl-[acyl-carrier-protein] reductase $[\mathrm{NADH}]$ from Mycobacterium tuberculosis with the ligand $5 \mathrm{e}$. The binding energy ranges from $-11.35 \mathrm{kcal} / \mathrm{mol}$ to $-7.1 \mathrm{kcal} / \mathrm{mol}$. The poses 1,7 and
9 had one hydrogen bond between the receptor, Enoyl-[acyl-carrier-protein]reductase [NADH] and ligand $5 e$, whereas conformations 5 and 8 had two hydrogen bonds. Among the ten different binding conformations, the conformation 5 with binding energy of $-9.95 \mathrm{kcal} / \mathrm{mol}$ and two hydrogen bonds is chosen as the best possible orientation which is displayed in Figure 5

The docking results of Enoyl-[acyl-carrierprotein] reductase [NADH] from Mycobacterium tuberculosis with the standard drug Isoniazid is presented in table $2 \mathrm{f}$. The binding energies of the different binding conformations range between -6.73 and $-5.83 \mathrm{kcal} / \mathrm{mol}$. Among the ten different docked conformations conformation 7 and 8 show 2 hydrogen bonds between the drug and Tyr158 and lle194. The conformation 1, Conformation 2, Conformation 3, Conformation 4 and Conformation 5 had only one hydrogen bond formed with binding energies -11.81, -10.68, -10.33, -9.82 and -9.56 $\mathrm{kcal} / \mathrm{mol}$ respectively. Hence, conformation 8 with 2 hydrogen bonds and binding energy of $-9.84 \mathrm{kcal} /$ $\mathrm{mol}$ is considered the best docked conformation and represented in Figure-6

Synthesized compounds were evaluated for antimycobacterial activity using Luciferase Reporter Phase (LRP) assay on MTB H37Rv and one isoniazid-resistant (INH-resistant) clinical isolate at National Institute for Research in Tuberculosis (NIRT), Chennai (28).

The percentage growth inhibition was recorded at the concentrations of 200, 100 and 50 $\mu \mathrm{g} / \mathrm{mL}$ (Table 3). Seeing the good inhibition of the synthesized compound further biological assays were conducted to determine their MIC values on different strains of Mycobacterium.

Results of these investigations indicate that some of the test compounds exhibited significant antimycobacterial activity along with antibacterial activity against all the tested strains.

\section{CONCLUSION}

A series of hydrazone Schiff base analogues were synthesized in good yield adopting standard procedures. The compounds were 
characterized by spectral studies. Docking studies of the above mentioned compounds revealed scope for antibacterial activity especially anti-tuberculosis activity. Further, all the study compounds exhibit a binding better than the existing drug isoniazid for Tuberculosis. This was further confirmed using in vivo studies where compound 4-chloro-benzoic acid (4-allyloxy-benzylidene)-hydrazide had maximum inhibition (97.9\%) $100 \mu \mathrm{mL}$. Hence, it can be concluded from the study that compound 4-chlorobenzoic acid (4-allyloxy-benzylidene)-hydrazide can be further evaluated for its efficiency in treating Tuberculosis.

\section{REFERENCES}

1. Ritesh P Bhole, Deepak. D. Borkar, Kishore. P. Bhusari and Prashant. A. Patil, Journal of Korean Chemical Society, 2012, 56(2) 236245

2. Liang Wang, Da-Gang Guo, Yan-Yan Wang and Chang-Zheng Zheng, RSC Adv., 2014, 4, 58895-58901

3. Vonburg R and Stout T, J. Appl. Toxicol., 1991, 11, 447-450

4. Chester E O, Norris G and Raymond D D, Anal. Profiles Drug Subt., 1979, 8, 283-314

5. Bedia K K, Elcin O, Seda U, Fatma K, Nathaly S, Sevim R and Dimogio A, Eur. J. Med. Chem., 2006, 41, 1253-1261

6. Immanuel $\mathrm{N} \mathrm{N}$, Ahmed $\mathrm{H}$, Felicite $\mathrm{M} \mathrm{M}$, innocent N N,Offiong $E O$ and Susan A B, Polyhedron, 2013, 63, 207-213

7. Kajal A, Bala S, Kamboj S and Saini V, Med. Chem. Res., 2014, 23, 2676-2689

8. Cikla P, Tatar E, Kucukguzel I, Sahin F, Yurdakul D, Basu A, Krishnan R, Nichols D B, Kaushik-Basu N and Kucukguzel S G, Med. Chem. Res., 2013, 22, 5685-5699

9. Yildirim LT and Atakol O, Cryst. Res. Technol., 2002, 37, 1352-1359

10. Yildirim L T, Emregul K C, Kurtaran R, and Atakol O, Cryst. Res. Technol., 2002, 37, 1344-1351

11. Bedia K K, Oruc- Emre E E, Unsalan S and Rollaas S, Med. Chem. Res., 2009, 18, 277286

12. Rosu, T., Negoiu, M., Pasculescu, S., Pahontu, E., Porier, D., Gulea, A., Eur. J.Med. Chem,2010, 45, 774-789

13. MahakSaini, Pradeep Kumar, Mahesh kumar, Kalavathy Ramasamy, Vasudevan Mani, Rakesh Kumar Mishra, Abu Bakar Abdul Majeed, Balasubramanian Narasimhan, Arab. J. of chem., 2014, 7, 448-460

14. Kucukguzel S G, Rollas S, Kucukguzel I, Kiraz
M, Eur. J.Med. Chem., 1999, 34, 1093-1100

15. Patole J, Sandbhor U, Padhye S, Deobagkar D N, Anson C E, Powell A, Bioorg. Med.Chem. Lett. 2003, 13, 51-55

16. Maccari R, Ottana R and Vigorita M G, Bioorg. Med.Chem. Lett. 2005, 15, 2509-2513

17. Cocco M T, Congiu C, Onnis V, Pusceddu M C, Schivo M L, logu A, Eur. J. Med. Chem.34, 1999, 1071-1076

18. N. Karali, A. Kocabalkanli, A. Gursoy, O. Ates, Farmaco, , 2002 , 57, 589-593

19. Rando D G, Santo D N, siqueira L, Malvezzi A, Leite C Q F, Amaral A T, Ferreira E I, Tavares L C, Boiorg. Med. Chem. 2002, 10, 557-560

20. Kocyigit-Kaymakcioglu Bedia, oruc Elcin, Unsalan Seda, Kandermirili Fatma, Shvets Nathaly, Rollas Sevim, Anatholy Dimoglo, Eur, j. Med. Chem.2006, 41, 1253-1261

21. Narender Malothu, Jaswanth S. Bhandaru, Umasankar Kulandaivelub, Malathi Jojula, Raghuram Reddy Adidala, Umadevi K. R., Dusthackeer A. V. N, Venkat Rao Kaki, Raghuram R. Akkinepally, Bioorganic \& Medicinal Chemistry Letters,2016, 26, 836-840

22. Monazza Serwar,Tashfeen Akhtar, Shahid Hameed and Khalid M. Khan, ARKIVOC 2009 (vii) 210-221

23. Langumin A, Stepanchikova Alla, Filimonov Dmitrii and Poroikov Vladimir, 2000, PASS: Prediction of activity spectra for biologically active substances, Bioinformatics, 16(8), 747748

24. F.C.Bernstein, T.F Koetzle, G.T.Williams, E.E.MeyerJr, M.D.Brice, J.R.Rodgers, O.Kennard, T.Shimanouchi, M.Tasumi, "The Protein Data Bank: A computer based Archival File for Macromolecular Structure" J of Mol.Biol., 1997, 112, 535.

25. Morris, GM, Huey R, Lindstorm W, Sanner 
MF, Belew RK, Goodsell, DS and Olson,AJ (2009), Autodock 4 and Autodock Tool 54: automated docking with selective receptor flexibility, J. Computational Chemistry, 2009, 16, 2785-2791.

26. Walker R.D.. Antimicrobial susceptibility testing and interpretation of results. In: Antimicrobial Therapy in Veterinary Medicine, Prescott J.F., Baggot J.D., Walker R.D., eds. Ames, IA, lowa State University Press, 2000.,12-26.

27. (a) Shawar, R. M.; Humble, D. J.; van Dalfsen,
J. M.; Stover, C. K.; Hickey, M. J.; Steele, S.; Mitscher, L. A.; Baker, W. Antimicrob. Agents Chemother.1997, 41, 570; (b) Prabu, A.; Seenivasan, P.; Kumar, V. Asian J. Med. Sci. 2014, 5, 54; (c) Dusthackeer, A.; Kumar, V.; Subbian, S.; Sivaramakrishnan, G.; Zhu, G.; Subramanyam, B.; Hassan, S.; Nagamaiah, S.; Chan, J.; Rama, N. P. J. Microbiol. Methods2008, 73, 18.

28. Tran, T.; Saheba, E.; Arcerio, A. V.; Chavez, V.; Li, Q.-Y.; Martinez, L. E.; Primm, T.P. Bioorg. Med. Chem. 2004, 12, 4809. 